\title{
CICLO DE VIDA DE RECURSOS ELETRÔNICOS: ENFOQUE NA GESTÃO DE COLEÇÕES EM BIBLIOTECAS UNIVERSITÁRIAS
}

\begin{abstract}
Resumo: Revisão que discorre sobre a formação e a gestão das coleções eletrônicas em bibliotecas universitárias, com base na apresentação de um modelo conceitual do ciclo de vida dos recursos eletrônicos de informação. A pesquisa bibliográfica abrangeu livros, artigos de revistas científicas, trabalhos apresentados em eventos, normas e documentos de organismos internacionais. Aborda a evolução histórica das coleções nas bibliotecas, sobretudo as universitárias, até a era das tecnologias, em que a insurgência do meio eletrônico culminou em transformações para a gestão dos acervos. Com base no ciclo de vida proposto, versa sobre aspectos da seleção, aquisição, acesso, gerenciamento, preservação e avaliação dos recursos eletrônicos. Aponta que os autores utilizados para a construção do modelo conceitual são unanimes em afirmar que o ciclo de vida dos recursos eletrônicos vem alterando o fluxo de trabalho nas bibliotecas, apesar de delinearem diferentes etapas para o ciclo e, portanto, entender o fluxo de trabalho sob outro prisma. E ainda se ressalta que compreender o ciclo de vida dos recursos eletrônicos de informação é fundamental para que os bibliotecários desempenhem efetivamente o seu papel no desenvolvimento científico universitário.
\end{abstract}

Palavras-chave: Recursos eletrônicos. Ciclo de Vida. Bibliotecas Universitárias.

\author{
Manoela Hermes Rietjens \\ Mestre em Gestão da Informação \\ Universidade do Estado de Santa \\ Catarina \\ manoelahermesr@gmail.com \\ Márcia Silveira Kroeff \\ Universidade do Estado de Santa \\ Catarina \\ Professora do Departamento de \\ Biblioteconomia \\ ms.kroeff@gmail.com \\ Liliane Vieira Pinheiro \\ Doutora em Ciência da Informação \\ Universidade Federal de Santa \\ Catarina \\ lilianepinheiro@gmail.com
}

\section{ELECTRONIC RESOURCES LIFE CYCLE: WITH ENPHASIS IN COLLECTION MANAGEMENT AT UNIVERSITY LIBRARIES}

\begin{abstract}
This revision addresses the development and management regarding to the electronic collections within the university libraries, based on the conceptual model presentation of the electronic information resources life cycle. The bibliographic research was covered by books, articles taken from scientific journals, works which were presented at conferences, norms and documents created and controlled by international entities. It indicates the historical evolution of the collections in the libraries, which goes from the first data found about the related subject, especially the one found at the universities, to the technologies Era, in which the electronic media insurgency culminated in transformations on the way the collections are managed. Based on the proposed life cycle, it relates on aspects of selection, acquisition, access, management, preservation and evaluation of electronic resources. It shows out that the authors are unanimous to assert that the life cycle of the electronic resources has been changing the workflow in the libraries, although they are delined in different stages for the cycle and therefore understand the workflow under another prism. And yet it must be emphasized that understanding the life cycle of electronic information resources is critical to the fact that librarians effectively may play their part accordingly to the university scientific development.
\end{abstract}

Key words: Electronic Resources. Life cycle. University Libraries. 


\section{INTRODUÇÃO}

As bibliotecas são instituições seculares e ao longo da história da humanidade foram as principais responsáveis pelo armazenamento e disponibilização do conhecimento registrado em variados suportes e formatos. Segundo McGarry (1999, p. 111), “[...] as bibliotecas, em seu sentido mais amplo, existem há quase tanto tempo quanto os próprios registros escritos." Por isso, a história das bibliotecas e das suas coleções acompanharam as transformações das sociedades a que serviam.

A história das bibliotecas e das suas coleções acompanharam as transformações das sociedades a que serviam, as tecnologias de informação e comunicação (TIC) vêm revolucionando substancialmente a forma como o conhecimento é registrado e disponibilizado ao público. No que se refere às bibliotecas, os recursos eletrônicos trazem novas configurações para a seleção, organização e disponibilização da informação.

McGarry (1999) apresenta as ferramentas da informação em cinco etapas: oral, alfabeto, manuscrito, tipografia e eletrônico, sendo que para o autor, tais etapas não eliminam umas às outras e podem coexistir em harmonia. $\mathrm{Na}$ atualidade, as bibliotecas estão cada vez mais trabalhando com coleções híbridas e as fontes de informação eletrônicas, ou recursos eletrônicos vem transformando a forma como as bibliotecas administram suas coleções.

Sabe-se que nas bibliotecas universitárias as coleções são formadas para atender principalmente os três pilares que compõem a universidade: ensino, pesquisa e extensão. Elas são vistas como as principais entidades gestoras de informações (BUENO DE LA FUENTE, 2010), seja servindo aos professores e alunos (PÉREZ ALARCÓN, 2001) ou disseminando o conhecimento científico desenvolvido na própria universidade. (NUNES; CARVALHO, 2016). Essas características requerem que estas instituições disponibilizem publicações nas diversas áreas do conhecimento, contemplando aspectos consolidados e atualizados do conhecimento, mesclando fontes de informação tradicionais com as novas formas em que a informação se apresenta.

Sob esta óptica o presente artigo visa discorrer sobre o fluxo de trabalho da gestão dos recursos eletrônicos em bibliotecas universitárias pelo prisma do ciclo de vida de tais recursos. 


\section{AS COLEÇÕES EM BIBLIOTECAS UNIVERSITÁRIAS}

A história das bibliotecas está ligada aos registros do conhecimento humano. Tais registros foram se modificando ao longo dos anos e, consequentemente, as coleções das bibliotecas acompanham as transformações adaptando-se.

A coleção é um dos principais componentes da biblioteca, entretanto, como os elementos que formam esta coleção vêm sendo modificados em decorrência da ascensão das tecnologias e das novas necessidades impostas pelos usuários, San José Montano (2011) entende que as coleções estão se transformando em um conjunto de recursos de conteúdo e informação ou ainda segundo Johnson (2014) podem ser vistas como a soma de todos os materiais, independente de formato e gênero que a biblioteca possui ou fornece acesso, por meio de compra ou locação.

Historicamente, os materiais utilizados para a escrita, tais como tabletas de madeira, folhas de palmeira, panos, seda, pergaminho e papel, foram suportes para o registro do conhecimento humano e possibilitaram a formação das coleções em bibliotecas (MARTINS, 2002).

$\mathrm{Na}$ Antiguidade, as bibliotecas eram constituídas por tabletas de argila, posteriormente por rolos de papiro ou pergaminhos, sendo que os manuscritos enrolados permaneceram até o ano 300, quando apareceram os códex por volta do século IV. Na Idade Média a circulação e manuseio do livro eram restritos ao ambiente clerical (MARTINS, 2002). Durante este período os monges estão associados às bibliotecas e são sempre representados no meio dos livros, seja os que copiam ou os que leem (BARATINI; JACOB, 2006). A Revolução Industrial, marca a transformação da tipografia artesanal em indústria, além de favorecer a democratização da cultura (MARTINS, 2002). Ocorre então uma explosão bibliográfica, marcada não apenas pelo número de exemplares disponíveis, mas também pela diversidade dos conteúdos publicados (MCGARRY, 1999). E quase cinco décadas após o ápice da explosão bibliográfica, o documento eletrônico impulsiona o novo modelo de biblioteca que se baseia no acesso e se legitima no advento da Internet (WEITZEL, 2002).

Atualmente, os recursos eletrônicos são uma parte inseparável das bibliotecas e dos pesquisadores. Os recursos eletrônicos são descritos como recursos publicados por meio de mídia eletrônica, que requerem o uso de equipamentos eletrônicos para acesso 
do seu conteúdo. São definidos como quaisquer publicações criadas digitalmente e armazenadas em formato eletrônico (ANBU K.; KATARIA; RAM, 2013, p. 300).

Weitzel (2002, p. 64) destaca que "a adoção das tecnologias da informação em bibliotecas, certamente revolucionou todos os processos e atividades bibliotecárias" e ao mesmo tempo em que os recursos eletrônicos trazem um desafio para o desenvolvimento de coleções também possibilitam uma oportunidade favorável se considerar-se a consagração do documento eletrônico.

O mesmo foi destacado por Vergueiro (1993), que aponta que a preocupação com o desenvolvimento das coleções ganhou força nas últimas décadas, após bibliotecários e gestores verificarem que seria impossível acompanhar o ritmo acelerado de expansão dos materiais informacionais e manter nas bibliotecas tudo o que era publicado, até porque seria inviável a construção de espaços físicos capazes de abrigar todo o conteúdo produzido e de realizar o devido tratamento técnico.

O desenvolvimento de coleções está atrelado à preocupação dos bibliotecários com as coleções e a necessidade de desenvolver, selecionar e desfazer acervos com o objetivo de tornar as bibliotecas mais coerentes, ou seja, a preocupação passou de simplesmente acumular material para torná-lo acessível (VERGUEIRO, 1989).

Pode-se compreender que "desenvolvimento de coleções é uma disciplina [da biblioteconomia] que procura organizar o conhecimento registrado sob enfoques e filtros específicos - uma solução técnica desencadeada pela explosão bibliográfica." (WEITZEL, 2002, p. 64). Esse processo, de acordo com Weitzel (2013) compreende algumas etapas: estudo da comunidade, política de seleção, seleção, aquisição e política de aquisição, desbastamento e política de desbastamento, avaliação e política de avaliação.

Os recursos das bibliotecas universitárias são reunidos principalmente em resposta às demandas de ensino e pesquisa (REDMOND; SINCHAIR; BROWN, 1972) e considerando a consolidação das informações eletrônicas, sobretudo dos periódicos científicos, pelos usuários (SAN JOSÉ MONTANO, 2011) associada a maior disponibilidade de informações na Web nas últimas décadas aumentaram as expectativas destes com relação ao acesso imediato a qualquer conteúdo que necessitam e assim as bibliotecas estão adquirindo cada vez mais recursos eletrônicos, modificando a forma como investem seus recursos financeiros (VERMINSKI; BLANCHAT, 2017). Atualmente as bibliotecas vêm investindo seus orçamentos de aquisição cada vez mais 
em recursos eletrônicos, deixando os impressos em segundo plano (VERMINSKI; BLANCHAT, 2017).

Embora os recursos eletrônicos sejam muito fáceis de usar e possuam um valor significativo de pesquisa para os pesquisadores, não há dúvida de que eles trazem entraves, mesmo depois de duas décadas do seu advento, aos administradores da biblioteca, uma vez que são difíceis de catalogar, administrar e gerenciar (ANBU K.; KATARIA; RAM, 2013).

A disponibilidade dos recursos eletrônicos traz desafios aos bibliotecários, acostumados, há séculos, com o armazenamento, organização e disseminação dos materiais impressos. Desta forma, também transformam a forma como as coleções são planejadas e formadas, trazendo novos elementos que precisam ser considerados no desenvolvimento das coleções, bem como para a gestão destas. Segundo Karmakar (2015) vários fatores tornaram o trabalho dos bibliotecários que lidam com recursos eletrônicos extremamente desafiadores, tais como: o incrível crescimento das coleções eletrônicas em bibliotecas, os grandes orçamentos envolvidos na sua aquisição, a variação infinita nos pacotes oferecidos pelas editoras, provedores de interface e agências de assinatura, as frequentes mudanças nos modelos de negócios e de preços e a falta de ferramentas automatizadas para lidar com a complexidade do gerenciamento de recursos eletrônicos.

O ciclo de vida da aquisição de recursos eletrônicos tornou-se mais complexo e segue um processo mais circular e iterativo do que o fluxo de trabalho que dominava o panorama da biblioteca (HOSBURGH, 2014). Considerando que existem diferenças significativas na gestão de recursos impressos e eletrônicos nas bibliotecas, sendo que a gestão dos recursos eletrônicos ainda apresenta muitas lacunas, dúvidas e desafios, cabe um estudo aprofundado sobre o fluxo de trabalho da gestão dos recursos eletrônicos em bibliotecas universitárias pelo prisma do ciclo de vida de tais recursos.

Para tal, baseou nos seguintes procedimentos metodológicos:

\section{CLASSIFICAÇÃO DA PESQUISA}

Trata-se de uma pesquisa bibliográfica que buscou aporte teórico na literatura publicada sobre o ciclo de vida de recursos eletrônicos, de modo a contribuir para a discussão da formação e gestão de coleções em bibliotecas universitárias. 
A pesquisa bibliográfica é considerada uma técnica para a obtenção dos dados (elementos críticos) e elaboração de resultados teóricos. A pesquisa bibliográfica é baseada nos materiais publicados e "[...] abrange toda bibliografia já tornada pública em relação ao tema de estudo" (MARCONI; LAKATOS, 2010, p. 166). Marconi e Lakatos (2010, p. 166) ainda ressaltam que “[...] a pesquisa bibliográfica não é mera repetição do que já foi dito ou escrito sobre certo assunto, mas propicia o exame de um tema sob novo enfoque ou abordagem, chegando a conclusões inovadoras".

Vale destacar que a revisão se encaminha para uma revisão narrativa, pois se utilizou de fontes aleatórias. Segundo Rother (2007, p. v), esse tipo de revisão é utilizado para “[...] descrever e discutir o desenvolvimento ou o 'estado da arte' de um determinado assunto, sob ponto de vista teórico ou conceitual [...]” e também “[...] não informam as fontes de informação utilizadas, a metodologia para a busca de referências, nem os critérios utilizados na avaliação e seleção dos trabalhos [...]". Com base nas publicações foi construído um modelo conceitual do ciclo de vida de recursos eletrônicos e ao longo do texto foram comparados os pontos de vistas dos diferentes autores que discutem sobre tal ciclo.

A pesquisa pode ser considerada também de cunho qualitativo e exploratório. É qualitativa por fundamentar-se na interpretação, pois conforme afirma Creswell (2007, p. 186-187), em uma pesquisa qualitativa “[...] o pesquisador faz interpretação dos dados[...]" e os filtra "[...] através de uma lente pessoal situada em um momento sociopolítico e histórico específico. Não é possível evitar as interpretações pessoais, na análise dos dados qualitativos." Creswell (2007) também destaca que uma das razões para a condução de um estudo qualitativo é sua característica exploratória.

Trata-se de uma pesquisa exploratória quanto aos objetivos por "proporcionar maior familiaridade com o problema, com vistas a torná-lo explícito” e também por “[...] ser bastante flexível [...]" e "[...] considerar os mais variados aspectos relativos ao fato ou fenômeno estudado." (GIL, 2010, p. 27).

\section{CICLO DE VIDA DE RECURSOS ELETRÔNICOS}

Os recursos eletrônicos apresentam peculiaridades e dinâmica diferente dos recursos tradicionais impressos, segundo Pesch (2008) há muito mais etapas no ciclo de 
vida de um recurso eletrônico quando em comparação com os impressos e muitas outras partes estão envolvidas. Na visão de Pesch (2008) afirmar que os recursos eletrônicos são desafiadores é um eufemismo. Embora a tecnologia ofereça mais acesso e possibilidades, também torna mais complexo o desenvolvimento da coleção de recursos digitais (FARMER, 2012).

As fontes de referência digitais merecem ser consideradas ao longo do seu ciclo de vida: da seleção ao processamento, passando pela manutenção e eventual cancelamento. As instituições precisam ter uma visão clara de suas necessidades e capacidades de informação, boas relações com os afiliados e boas habilidades de negociação com os fornecedores (FARMER, 2012). Em consonância com o exposto, Karmakar (2015) ressalta que o ciclo de vida dos recursos eletrônicos e os desafios enfrentados pelos bibliotecários precisam ser examinados na gestão dos recursos.

O quadro a seguir apresenta as etapas dos ciclos de vidas dos recursos eletrônicos na visão de diferentes autores, utilizados como referências para a proposição do modelo conceitual, no presente estudo.

Quadro 1 - Etapas do ciclo de vida dos recursos eletrônicos, por autor

\begin{tabular}{|c|c|}
\hline AUTOR & ETAPAS \\
\hline JEWELL (2001) & $\begin{array}{l}\text { Selecionar; Procedimentos internos de avaliação e compra; } \\
\text { Questões de licenciamento; Apresentação na Web; Suporte de } \\
\text { uso; Avaliação contínua e informações de uso; Preservação e } \\
\text { arquivamento. }\end{array}$ \\
\hline PESCH $(2004,2008)$ & $\begin{array}{l}\text { Adquirir; Prover acesso; Administrar; Prover suporte; Avaliar e } \\
\text { monitorar. }\end{array}$ \\
\hline $\begin{array}{l}\text { SADEH, ELLINGSEN } \\
(2005)\end{array}$ & $\begin{array}{l}\text { Descobrir; Trial; Selecionar; Adquirir; Acessar; Decidir pela } \\
\text { renovação ou cancelamento. }\end{array}$ \\
\hline FARMER $(2009,2012)$ & $\begin{array}{l}\text { Pré-planejamento; Aquisições; Licenciamento; Apresentação na } \\
\text { web; Uso; Manutenção; Conservação; Arquivamento e revisão. }\end{array}$ \\
\hline EMERY; STONE (2013) & $\begin{array}{l}\text { Investigação de novos conteúdos; Aquisição de novos } \\
\text { conteúdos; Implementação; Revisão e acesso; Revisão anual; } \\
\text { Cancelamento e revisão para substituição. }\end{array}$ \\
\hline $\begin{array}{l}\text { ANBU K.; KATARIA E } \\
\text { RAM (2013) }\end{array}$ & Utiliza o modelo de Sadeh e Ellingsen (2005). \\
\hline HOSBURGH (2014) & Utiliza o modelo de Emery e Stone (2013). \\
\hline KARMAKAR (2015) & $\begin{array}{l}\text { Adquirir; Prover acesso; Administrar; Prover suporte; Avaliar e } \\
\text { monitorar. }\end{array}$ \\
\hline GEUTHER (2017) & Utiliza o modelo de Pesch (2008) \\
\hline
\end{tabular}

Fonte: dados da pesquisa, 2018. 
A figura 1 apresenta o ciclo de vida dos recursos eletrônicos de informação adaptado pelas pesquisadoras, com base em Jewell (2001), Pesch (2004, 2008), Sadeh e Ellingsen (2005), Farmer (2009, 2012), Emery e Stone (2013), Anbu k.; Kataria e Ram (2013), Hosburgh (2014), Karmakar (2015) e Geuther (2017).

Figura 1 - Ciclo de vida dos recursos eletrônicos

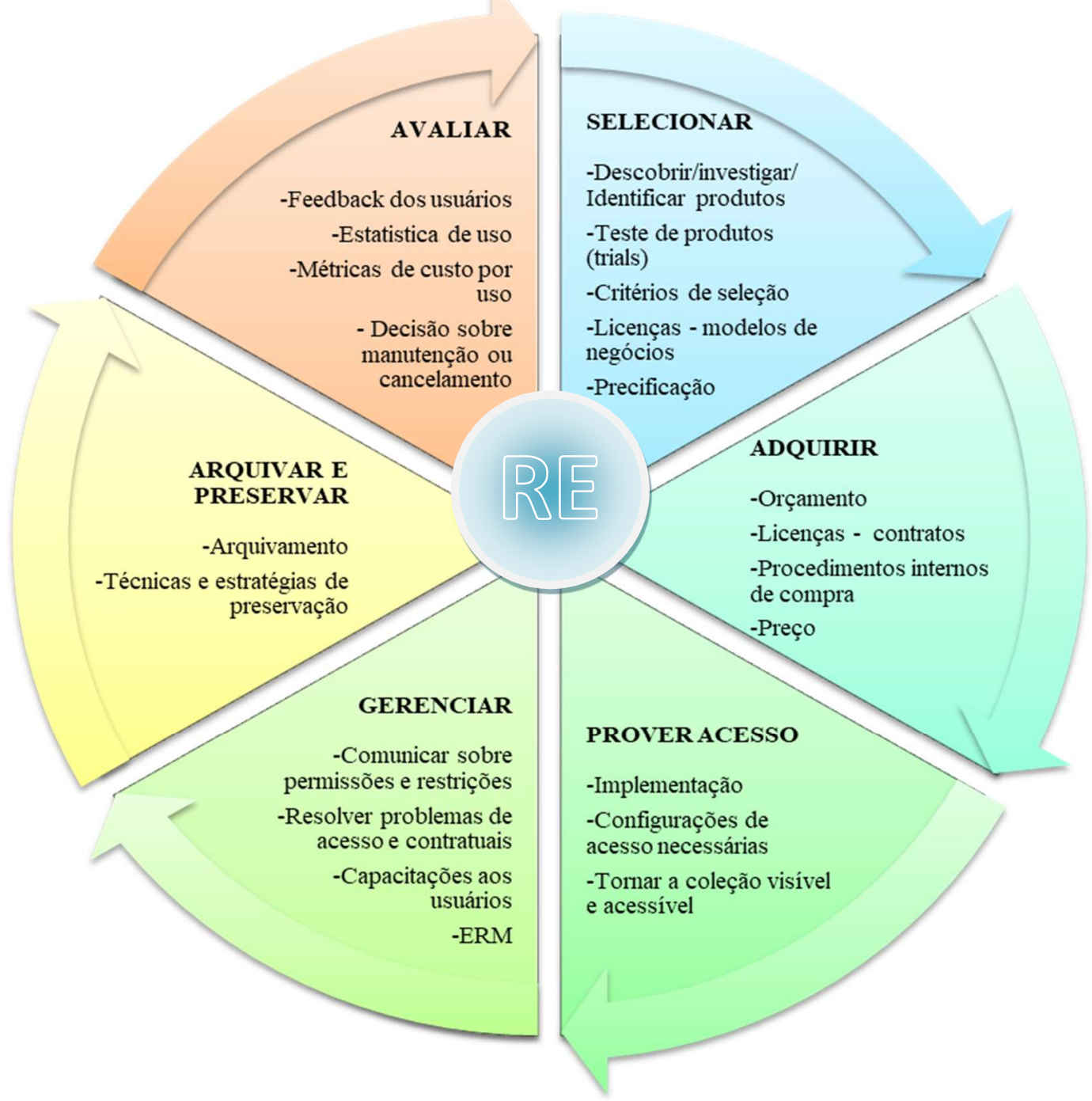

Fonte: Adaptado de Pesch (2004, 2008), Sadeh e Ellingsen (2005), Farmer (2009, 2012), Emery e Stone (2013), Anbu k.; Kataria e Ram (2013), Hosburgh (2014), Karmakar (2015) e Geuther (2017). 


\subsection{AS ETAPAS DO CICLO DE VIDA DE RECURSOS ELETRÔNICOS NA GESTÃO DE COLEÇÕES EM BIBLIOTECAS UNIVERSITÁRIAS}

A gestão dos recursos eletrônicos exige dos responsáveis pela coleção, uma série de conhecimentos, atividades e atitudes diferenciadas, algumas das quais, não eram necessárias para as obras impressas. Para a melhor compreensão do que se fala, descrevese a seguir cada uma das seis etapas do ciclo de vida dos recursos eletrônicos, elencadas na figura 1.

\subsubsection{Selecionar}

A seleção envolve a tomada de decisão sobre a inclusão de recursos na coleção da biblioteca e na visão de Anbu K, Kataria e Ram (2013) pode iniciar com a descoberta e identificação do recurso, aspectos que, para os autores, são os mais importantes no processo de gerenciamento de recurso eletrônico. Já Hosburgh (2014), para este processo, usa o termo investigar.

A identificação do recurso para uma instituição acadêmica pode ter várias fontes, tais como a faculdade, o bibliotecário, um anúncio on-line, um alerta encaminhado por email, o editor ou a busca por um conteúdo substancial. A descoberta/investigação/identificação abre caminho para o resto do ciclo de vida dos recursos eletrônicos (ANBU K.; KATARIA; RAM, 2013).

Farmer (2009, p. 118) alerta que existe, uma cobrança da comunidade acadêmica com relação à disponibilização da informação eletrônica pelas bibliotecas, o que muito frequentemente, leva os profissionais a refletirem sobre as práticas de gestão das coleções, de forma a possuir “[...] uma atitude reativa, e não uma postura estratégica responsável”.

Portanto, é oportuno que a biblioteca tenha claro suas intenções para a coleção que pretende oferecer à comunidade. Nesse sentido, Hosburgh (2014) ressalta que ao descobrir/investigar/identificar novos conteúdos é importante saber o que se quer alcançar. Para o autor, é fundamental aferir a demanda e o propósito, isto é, se o recurso atenderá professores e alunos, se é destinado ao ensino, pesquisa ou desenvolvimento profissional, bem como medir o potencial para uso futuro.

Para chegar a esse nível de clareza a respeito da adequação do recurso analisado ao planejamento estratégico da biblioteca, existem elementos a serem considerados que 
podem impulsionar a tomada de decisão baseadas em uma série de critérios envolvidos na seleção dos recursos (HOSBURGH, 2014), tais como escopo, arranjo, facilidade de uso, propósito, público alvo, autoridade, interface, legibilidade, transferência de arquivos, requisitos técnicos e acordos de licenciamento, iniciativas de código aberto (FARMER, 2012). Os bibliotecários devem levar em conta ainda a missão da biblioteca; necessidades de seus usuários; questões de linguagem, de acessibilidade, de administração de software, como segurança, autenticação; espaço e equipamentos, infraestrutura técnica, coleções atuais, disponibilidade de compartilhamento de recursos e custos (FARMER, 2012).

A International Federation of Library Associations and Institutions ${ }^{1}$ (2012) define outros critérios que devem ser analisados no momento de escolher determinado recurso, como por exemplo, o método de acesso, a forma de autenticação, a compatibilidade com hardwares, softwares, navegadores e formatos de conteúdo, a interface, como ocorre a pesquisa e a recuperação, o oferecimento de registros de catalogação, se permite exportações, downloads e integração com outras plataformas, como se dá o suporte do fornecedor, licenciamento e modelo de negócios, qual o número de utilizadores, entre outros.

Dessa forma, é fundamental que as bibliotecas definam seus escopos de atuação, conheçam profundamente as tecnologias que possuem a sua disposição bem como os interesses da comunidade que atendem. Um dos documentos que ajuda a cumprir essa função estratégica e que está à disposição das bibliotecas é a política de desenvolvimento de coleções. Por isso, Emery e Stone (2013) afirmam ser fundamental definir os critérios que se deseja cumprir e mapeá-los nesta política. Entretanto, sobre o assunto, Farmer (2009, p. 118, tradução nossa) ressalta que “[...] as políticas de coleções de bibliotecas às vezes ficam para trás da curva de tecnologia." O que demonstra que os documentos normativos muitas vezes não preveem ou consideram recursos eletrônicos em seus textos.

$\mathrm{Na}$ opção pela seleção de recursos eletrônicos em detrimento de impressos é indispensável o conhecimento a respeito da comunidade a ser atendida e ainda, conforme Farmer (2009), conhecimento aprofundado da coleção já existente. Além disso, são fundamentais processos de seleção de recursos eletrônicos bem estruturados, sendo que uma sugestão é a inclusão de um checklist que auxilie a verificar cada característica dos

\footnotetext{
${ }^{1}$ Federação Internacional das Instituições e Associações Bibliotecárias. É uma organização internacional de bibliotecas que representa os interesses de bibliotecas, serviços de informações e seus usuários (INTERNATIONAL FEDERATION OF LIBRARY ASSOCIATIONS AND INSTITUTIONS, 2012).
} 
recursos eletrônicos (INTERNATIONAL FEDERATION OF LIBRARY ASSOCIATIONS AND INSTITUTIONS, 2012).

Karmakar (2015) entende que a aquisição de um recurso eletrônico geralmente envolve a organização de testes, a revisão e a negociação de um contrato de licença e a compreensão e o aceite de modelos complexos de preços, entretanto, neste estudo utilizarse-á a visão proposta pela International Federation of Library Associations and Institutions (2012), que entende estes passos como inerentes a atividade de seleção.

A respeito dos modelos de compra e preços, a International Federation of Library Associations and Institutions (2012, p. 11) salienta que “[...] não há um modelo padrão para recursos eletrônicos", como por exemplo, podem apresentar preços separados para conteúdos e acesso, preços de pacote, modelos de preços orientados ao cliente, pagamento por utilização, entre outros. Portanto, no momento da seleção é essencial a análise cuidadosa de todos os modelos disponíveis no mercado, decidindo por aqueles que melhor atenderem a missão da biblioteca em questão.

Ressalta-se que, nos ciclos de vida propostos por Farmer (2009) e Pesch (2004), as questões inerentes à licenciamento de recursos eletrônicos encontravam-se posteriores à seleção, entretanto, acredita-se que as licenças também são quesitos decisivos no processo de escolha por determinado recurso, concordando com a International Federation of Library Associations and Institutions (2012) e por isso, optou-se por deixála atrelada, tanto na seleção, quando se opta por incluir o recurso na coleção com determinado modelo de negócios, quanto na aquisição, quando se efetiva as questões contratuais de licenciamento.

Dessa forma, os períodos de testes (trials) são relevantes para que se experimente e se faça uma avaliação prévia do produto, sendo importante incluir a comunidade acadêmica nesta avaliação, e para isso se faz necessária a divulgação do potencial recurso para que os usuários verifiquem também o seu valor (SADEH; ELLINGSEN, 2005; FARMER, 2009; INTERNATIONAL FEDERATION OF LIBRARY ASSOCIATIONS AND INSTITUTIONS, 2012). O tempo e a duração do trial também são importantes, bem como a sua divulgação, que pode utilizar-se de canais como blog, wiki, web, e-mail ou até mesmo o boca-a-boca (HOSBURGH, 2014).

É conveniente, portanto, que as bibliotecas usufruam dos períodos de trials, pois são eles que permitem a aproximação da comunidade à satisfação da sua necessidade de informação. Vale ressaltar ainda a importância da participação, nesta avaliação, de 
especialistas na área, preparados para julgar a qualidade dos conteúdos disponibilizados e de bibliotecários especialistas em assuntos e conhecedores das características dos usuários, capazes de analisar os recursos adicionais das fontes de informação, na intenção de testar o seu alinhamento com as necessidades de pesquisa da instituição.

Destaca-se ainda a pertinência durante o processo de seleção de se verificar cuidadosamente a sobreposição de cobertura de conteúdo entre os fornecedores (FARMER, 2009, 2012; HOSBURGH, 2014). É comum que os agregadores de banco de dados agrupem vários recursos e por isso é desejável que a sobreposição da cobertura seja analisada juntamente com as particularidades de cada serviço oferecido na linha de produtos do fornecedor (FARMER, 2012).

Essa verificação é indicada para que não se duplique conteúdo, lembrando-se de considerar tanto conteúdos já disponibilizados na biblioteca, independente dos formatos, quanto novos possíveis conteúdos a serem adicionados. Ressalva-se ainda que sobrepor conteúdo é investir recursos financeiros em duplicidade, o que recomenda-se evitar.

Segundo Hosburgh (2014), os bibliotecários não devem ser tímidos em informar aos fornecedores que estão verificando as condições favoráveis e preços de produtos similares. Também é desejável questionar sobre a disponibilidade de acordos de consórcios como uma alternativa a redução de custos. Fica implícita assim a necessidade do domínio dos bibliotecários com relação à coleção da biblioteca e às ofertas existentes no mercado, empoderando o profissional frente aos fornecedores, agregando capacidade de negociação e possível conquista de melhores acordos.

A respeito das questões relativas ao preço, entende-se que pode ser um indicador considerado tanto na seleção, quanto na aquisição (INTERNATIONAL FEDERATION OF LIBRARY ASSOCIATIONS AND INSTITUTIONS, 2012; HOSBURGH, 2014).

Em se tratando da precificação, além das características previstas nos modelos de negócios, é vantajoso basear-se no método de mensuração equivalente de tempo integral (FTE), de modo a considerar quem estará realmente utilizando o recurso (HOSBURGH, 2014).

A análise de tais elementos embasa a tomada de decisão do bibliotecário que seleciona recursos eletrônicos, pois permite o estudo de todos os critérios, desde os tradicionais até os exclusivos, optando assim pelo recurso que estiver mais alinhado com os objetivos institucionais e a necessidades do público-alvo. 


\title{
4.1.2 Adquirir
}

Após verificar todas as questões inerentes às características do recurso eletrônico e selecioná-lo para aquisição, o passo seguinte é a concretização da aquisição. Para isso, é necessário, além dos trâmites que envolvem os processos de compra, com características particulares em cada instituição, verificar o preço do produto em relação ao orçamento da Biblioteca, além de analisar e modificar os acordos de licença e providenciar a fatura para pagamento (GEUTHER, 2017).

De acordo com Hosburgh (2014), na etapa da aquisição é imperativo verificar se a biblioteca e o fornecedor estão de acordo com todos os critérios estabelecidos anteriormente, bem como se é necessária uma ordem de compra, um contrato específíco e quais os termos do processo anual de renovação.

Como comumente os recursos eletrônicos envolvem acordos de licença, Farmer $(2012,2009)$ destaca a complexidade destes empreendimentos, uma vez que se lida com questões como direitos autorais e requisitos técnicos, sendo que o fornecedor pode limitar seriamente o uso dos recursos. Por isso o autor faz uma analogia interessante:

\begin{abstract}
Assim como os indivíduos podem se concentrar em comprar um carro de um revendedor que oferece um ótimo preço, mas esquecem de pensar se o revendedor dará o suporte necessário para o carro mais tarde, os bibliotecários acadêmicos também podem esquecer os problemas de manutenção e cancelamento (FARMER, 2009, p. 118, tradução nossa).
\end{abstract}

Dessa forma, torna-se fundamental que os bibliotecários revisem e negociem os acordos de licenças, pois “[...] a imagem real dos recursos de referência eletrônica não é tão simples quanto conectar um computador e clicar na conexão com a Internet" (FARMER, 2009, p. 118, tradução nossa). A International Federation of Library Associations and Institutions (2012, p. 13), salienta que "antes de comprar, a licença deve ser revista e negociada para informar e apoiar o processo de avaliação, e para garantir que ele reflete a expectativa do selecionador."

Durante a negociação do contrato existem várias considerações a serem feitas e é relevante verificar algumas licenças tradicionais que o fornecedor pode seguir, a exemplo do Shared Electronic Resource Understanding (SERU) ${ }^{2}$, citado por Hosburgh

\footnotetext{
${ }^{2}$ Compreensão Compartilhada de Recursos Eletrônicos.
} 
(2014). Assim sendo, uma negociação equilibrada entre bibliotecários e fornecedores evita o atraso na disponibilização do recurso e o SERU - resultado de um grupo de trabalho nos Estados Unidos da América - é uma declaração que pode ser utilizada como alternativa a um contrato de licença, expressando entendimentos compartilhados do provedor de conteúdo, da instituição assinante, dos usuários autorizados, além de mencionar questões como: a natureza do conteúdo, os usos inadequados, a privacidade e confidencialidade, o desempenho online, a prestação de serviços, o arquivamento e o acesso perpétuo (HAHN, 2007). Outra opção é a LIBLICENSE que fornece um modelo para bibliotecários utilizarem na negociação de acordos de licenciamento específicos e serve como uma declaração da comunidade de bibliotecas acadêmicas sobre o que considera política e prática aceitáveis para licenciar informações digitais (LIBLICENSE, 2015).

No Brasil, desconhecem-se iniciativas neste sentido e por isso, elencam-se alguns pontos citados pela International Federation of Library Associations and Institutions (2012) que devem ser abordados nos contratos: a) quem serão os utilizadores autorizados; b) a forma de acesso; c) a política de arquivamento; d) o uso que se pode fazer das informações eletrônicas (empréstimo entre bibliotecas, responsabilidade pelo uso não autorizado, visualização, impressão e download, confiabilidade das informações do utilizador); e) o suporte do fornecedor (disponibilidade de acesso, dados de catalogação, data de início), f) questões de rescisão ou cancelamento; g) condições de pagamento; h) jurisdição legal, entre outros.

Com relação ao preço na aquisição, Hosburgh (2014) afirma que é importante solicitar, com base nas análises de sobreposição de conteúdo (realizadas na seleção), ao fornecedor um ajuste no preço do recurso considerando o percentual de conteúdo que já está coberto por outros recursos existentes na biblioteca. O mesmo autor destaca que tudo é negociável.

\subsubsection{Prover acesso}

Ao contrário dos recursos em formato impresso cujo acesso ocorre ao catalogálo, classificá-lo e colocá-lo na estante, o fornecimento de acesso a recursos eletrôncios pode ser muito mais complexo, uma vez que o conteúdo geralmente não é armazenado na biblioteca (KARMAKAR, 2015). Assim, após o licenciamento do recurso eletrônico é 
necessário tanto configurar o acesso aos utilizadores autorizados, quanto tornar os recursos acessíveis no catálogo ou na página da biblioteca.

Geuther (2017) elenca as tarefas que compõem prover acesso como coordenar as configurações de IP e proxy com o fornecedor, adicionar espaço no servidor local, ativar o serviço de descoberta, efetuar o registro dos acervos e organizar a divulgação na página da biblioteca.

Já para Hosburgh (2014), o provimento do acesso é visto como parte da etapa de implementação, que na sua visão se inicia com o teste do produto, para certificar-se de que o Localizador Uniforme de Recursos (URL) é estável e aponta para a interface apropriada. Para ter certeza de que o acesso está ativado podem ser feitas verificações periódicas para verificar os links em vários pontos de acesso e via autenticação remota, verificar texto completo, assim como para problemas gerais de usabilidade que podem surgir quando se navega no site da biblioteca através da plataforma de um provedor.

Karmakar (2015) salienta que muito trabalho é dedicado ao gerenciamento de links para o conteúdo, de modo a auxiliar os usuários finais a descobrir o conteúdo através das listas, resolvedores de links ou do catálogo da biblioteca; e também ao gerenciamento da autenticação para garantir que os usuários autorizados possam acessar o conteúdo, o que pode ser feito registrando endereços IP com o editor ou por meio de servidores de autenticação e proxy do campus.

As questões técnicas de acesso, como protocolos de rede, procedimentos de autorização e autenticação, garantias de segurança, entre outros pontos, normalmente devem ser verificadas com o ramo técnico da instituição, entretanto, o North American Serials Interest Group estabelece que os bibliotecários devem possuir conhecimentos teóricos e práticos com relação a provisão de acesso à recursos eletrônicos (FARMER, 2009; NORTH AMERICAN SERIALS INTEREST GROUP, 2016).

Em relação aos periódicos eletrônicos é desafiante para as bibliotecas apresentálos na coleção e torná-los acessíveis, visto que muitas vezes as URL's disponibilizadas pelo fornecedor não direcionam ao título imediatamente e sim a um agregador. Uma alternativa é a elaboração de listas HTML, com todos os títulos disponíveis em lista alfabética e organizadas por assunto, a criação de um banco de dados local que armazene os dados para a geração de uma lista HTML pode otimizar o trabalho de manter a lista atualizada manualmente (MONTGOMERY; SPARKS, 2000). 
Alguns fornecedores oferecem registros bibliográficos no formato apropriado para entrada no OPAC $^{3}$ da biblioteca (INTERNATIONAL FEDERATION OF LIBRARY ASSOCIATIONS AND INSTITUTIONS, 2012). No entanto, cada vez mais, as bibliotecas estão incorporando mecanismos que servem como um único ponto de acesso à fonte desejada, seja física ou virtual, isto é, sistemas de descobertas, que são ferramentas que cobrem todos os pontos de acesso da biblioteca e que canalizam todo o conteúdo em uma única interface de busca (FARMER, 2012; VERMINSKI, BLANCHAT, 2017). Em contrapartida, alguns autores apontam empecilhos para a existência de um catálogo unificado, como Montgomery e Sparks (2000) que indicam a falta de URL's permanentes nas coleções de agregadores e, Verminski e Blanchat (2017) que mencionam a forma com que editores e fornecedores projetam suas plataformas para recuperação da informação.

Deste modo, estão implícitos os desafios e instabilidades que os bibliotecários encontram no provimento dos recursos eletrônicos, bem como as novas competências essenciais ao profissional, como a habilidade tecnológica, que aliada a antigos saberes, como o mapeamento avançado das características dos seus usuários e o domínio das coleções e ferramentas disponíveis, garantem uma disponibilização assertiva dos recursos de informação.

Diante do exposto, prover acesso significa tanto configurar as permissões de acesso a fonte de informação, como a sua disponibilização nos canais de divulgação que a biblioteca dispõe, sendo que a divulgação das fontes e a garantia do seu uso ainda são temas a serem tratados no próximo tópico, que diz respeito ao gerenciamento da coleção.

\subsubsection{Gerenciar}

A aquisição de fontes de referência digital não garante seu uso ideal. Os acessos eletrônicos devem ser organizados e gerenciados para recuperação e uso efetivos (FARMER, 2012). Após a disponibilização do recurso eletrônico na biblioteca, surge a necessidade de informar o usuário sobre sua existência, garantir a sua correta utilização, assim como favorecer o seu uso.

\footnotetext{
${ }^{3}$ Catálogo de acesso público online. A biblioteca de interface eletrônica usada pelos usuários para pesquisar ou navegar pelo conteúdo de um banco de dados, descrevendo uma coleção de bibliotecas. (CONGER, 2004). 
A divulgação é vista por Hosburgh (2014) como parte da etapa de implementação, para o autor dependendo do recurso eletrônico e da biblioteca pode ser necessário um plano de marketing. Este plano deve cobrir todas as necessidades e interesses de todos os usuários em potencial que podem precisar saber do recurso adquirido e ainda considerar os grupos de colaboradores da biblioteca ou administradores de pesquisa (EMERY; STONE, 2013).

Como uma maneira de informar os usuários sobre a disponibilidade do recurso a homepage da biblioteca pode ser um instrumento de divulgação, assim, a biblioteca destaca em sua página, ou onde vincula o recurso com informações, como ocorre o acesso, quais as configurações tecnológicas necessárias, bem como as permissões e restrições existentes no acordo de licença negociado. É recomendado como uma boa prática pela National Information Standards Organization (2012) que a instituição, mesmo que não possa controlar o comportamento dos usuários, tome todas as medidas cabíveis para informá-los sobre as condutas apropriadas e limite o acesso somente aos autorizados.

Ainda para instruir os usuários sobre a disponibilidade e formas de utilização do recurso, a equipe da biblioteca deve estar informada a respeito da existência do recurso e conhecer como se dá a utilização do mesmo, por isso é importante que o fornecedor ofereça uma formação inicial e contínua (INTERNATIONAL FEDERATION OF LIBRARY ASSOCIATIONS AND INSTITUTIONS, 2012). Normalmente, este treinamento oferecido pelos fornecedores se dá em forma de seminários online, teleconferências, podcasts ou instrução local (EMERY; STONE, 2013).

É fundamental ainda, mesmo que o recurso seja de fácil utilização, o fornecimento de orientações para os usuários, que podem ocorrer por meio da disponibilização de guias ou manuais online que expliquem a utilização do produto, elaboração de páginas wikis, blogs, podcasts, além de webinars gratuitos (HOSBURGH, 2014; EMERY; STONE, 2013; INTERNATIONAL FEDERATION OF LIBRARY ASSOCIATIONS AND INSTITUTIONS, 2012). Esses documentos podem ser produzidos pela biblioteca ou podem ser oferecidos pelo fornecedor (HOSBURGH, 2014).

Considerando que o acesso aos recursos eletrônicos comumente ocorre por intermédio da rede, podem transcorrer problemas que impeçam sua utilização e neste caso caberá aos bibliotecários gerenciar essas questões para evitar a interrupção do serviço (KARMAKAR, 2015). A National Information Standards Organization (2012) destaca 
que, caso sejam problemas contratuais, convém que tanto o fornecedor quanto a biblioteca tomem todas as medidas cabíveis para resolver qualquer incidente, o mais rapidamente possível.

Assinala-se, nesse sentido, que os recursos eletrônicos exigem uma equipe sinérgica, ou seja, como todos os profissionais que atuam nas bibliotecas são impactados pela decisão da inclusão na coleção de uma fonte de informação em meio eletrônico, deve haver engajamento e fluidez nas ações que são tomadas, mesmo que cada uma delas esteja ligada a diferentes departamentos.

Caso a biblioteca ofereça uma grande quantidade de recursos eletrônicos, um desafio para a equipe também é administrar a imensidão de informações relativas a cada um dos recursos, já que cada um deles apresenta um fornecedor, uma forma de acesso, questões de licenciamento próprias, bem como a necessidade de diferentes ações por parte da biblioteca.

Karmakar (2015) afirma que o ciclo de vida dos recursos eletrônicos evidencia a enormidade da tarefa do bibliotecário para gerenciá-los. A tarefa torna-se complicada devido às centenas de recursos eletrônicos adicionados e por não ser possível para as bibliotecas fornecerem acesso a todos os títulos de recursos eletrônicos por meio de seus catálogos. Por isso o mesmo autor aconselha manter o controle dessas informações.

A imensa quantidade de dados, juntamente com a incapacidade de significativamente converter as métricas, especialmente a análise de custos e as estatísticas de usos, levou os bibliotecários a procurar soluções melhores para o gerenciamento de recursos eletrônicos (ANBU K.: KATARIA; RAM, 2013). Pensando em resolver questões administrativas dos dados dos recursos eletrônicos, surgem, no início dos anos 2000, os Electronic Resources Management Systems (ERMS), ou sistemas de gerenciamento de recursos eletrônicos, com a promessa de auxiliar no compartilhamento de informações e no acompanhamento do ciclo de vida dos recursos eletrônicos (VERMINSKI; BLANCHAT, 2017).

ERMS podem ser entendidos como sistemas que suportam o gerenciamento das informações e do fluxo de trabalho necessários para selecionar, adquirir, manter e fornecer acesso aos recursos eletrônicos de acordo com os sistemas de negócios e os termos da licença (ANBU K.: KATARIA; RAM, 2013). Para complementar, a maioria dos sistemas de gerenciamento de recursos eletrônicos possibilita registrar informações sobre recursos eletrônicos, seus fornecedores, termos e condições, uso, custos, etc; 
ferramentas para tomada de decisão e suporte; códigos e alertas para ações de rastreamento; contatos; relatório de incidentes; processo e fluxo de trabalho baseados no ciclo de vida e consolidação de dados de uso de vários sites de conteúdo (KARMAKAR, 2015).

Alguns autores evidenciam a utilidade dos ERMS no controle dos recursos eletrônicos ao longo do seu ciclo de vida, como Hosburgh (2014) que afirma ser benéfico empregar uma abordagem de equipe neste gerenciamento e exercitar o controle de qualidade; e Karmakar (2015) que indica que o seu uso pode abrir novos horizontes relacionados à coleta de informações no mercado global, bem como servir de ferramenta para o pessoal da biblioteca.

Como curiosidade, existem algumas iniciativas opensource de ERMS, como o CORAL e CUFTS, que são citadas por Emery e Stone (2013). Já Minchew e Slutskaya (2016) citam outras ferramentas que vêm sendo utilizadas para apoiar o gerenciamento de recursos eletrônicos, como por exemplo, o sistema de gerenciamento de banco de dados Microsoft Access, o sistema de gestão de conteúdos LibGuide e Wikis e ferramenta de gestão de projetos Trello.

Independente da solução utilizada para resolver o fluxo de trabalho gerado pelos recursos eletrônicos, Verminski e Blanchat (2007) alertam que para obter sucesso no gerenciamento existe a exigência de uma correta inserção de dados por parte da equipe da biblioteca, como por exemplo, associar o recurso ao fornecedor, inserir o preço, relacionar informações de contrato, questões relacionadas a estatísticas de uso, bem como manutenção contínua dessas informações.

Percebe-se assim, que a insurgência dos recursos eletrônicos de informação cria, para a Biblioteconomia, novos processos a serem executados como também a necessidade da utilização de novas ferramentas e até mesmo sistemas, além dos gerenciadores de catálogos, comumente utilizados.

\subsubsection{Arquivar e preservar}

Com a coleção impressa há um compromisso não declarado (exceto certas regras de coleção) de manter o item para a posteridade, mesmo nas menores instituições (FARMER, 2012). Entretanto a preocupação com o arquivamento e preservação dos 
materiais adquiridos pela biblioteca, apesar de continuarem a existir com os recursos eletrônicos, é muito diferente da que se tem com os materiais impressos.

Particularmente, se o acesso a recursos eletrônicos for por assinatura é provável que ocorra uma reconsideração mais frequente. Se a comunidade não usar o recurso dentro do período de licenciamento, esse recurso poderá não ser renovado. Se, por outro lado, as instituições desejarem manter suas referências eletrônicas, elas devem considerar essa opção quando entrarem nas negociações, a fim de determinar se a instituição tem o direito de manter os dados (FARMER, 2012).

Trata-se sempre de uma questão de negociação com o fornecedor, que poderá manter acesso aos conteúdos adquiridos de forma perpétua, o que pode vir a envolver inclusive, a cobrança de uma taxa anual para continuar a oferecer acesso mesmo após o término da assinatura e para manter cópias de arquivo (NATIONAL INFORMATION STANDARDS ORGANIZATION, 2012). Essa alternativa é particularmente atraente para grandes instituições que desejam manter coleções completas de periódicos para seus pesquisadores (FARMER, 2012).

Considerando que o recurso eletrônico licenciado ou em acesso aberto não poderá ser mantido no espaço físico da biblioteca, é importante compreender a política de arquivamento do fornecedor do recurso, sendo que é recomendável que preveja um acordo com LOCKSS $^{4}$, Pórtico ${ }^{5}$ ou qualquer outro produto de preservação digital. (INTERNATIONAL FEDERATION OF LIBRARY ASSOCIATIONS AND INSTITUTIONS, 2012).

O projeto LOCKSS, uma iniciativa de várias instituições, pode contribuir com este processo de arquivamento. Assim como em outras decisões na gestão de coleções digitais de referência, a cooperação entre instituições pode ser a melhor solução (FARMER, 2012).

É indicado ainda que as instituições possuam um planejamento para possíveis casos de desastres naturais ou intencionais, uma vez que os ativos eletrônicos são surpreendentemente vulneráveis a violações de segurança, problemas elétricos, desmagnetização, água, calor e até "chamas" solares que podem derrubar os serviços. Os

\footnotetext{
${ }^{4}$ Lots of Copies Keeps Stuff Safe (Muitas cópias mantém as coisas seguras). É um método de armazenamento que se concentra em redundância de backup de informação, fornecendo vários servidores de armazenamento que oferece as bibliotecas e editores ferramentas de preservação com baixo custo e código aberto (INTERNATIONAL FEDERATION OF LIBRARY ASSOCIATIONS AND INSTITUTIONS, 2012).

${ }^{5}$ Serviço de preservação digital e arquivamento eletrônico da entidade sem fins lucrativos Ithaka (INTERNATIONAL FEDERATION OF LIBRARY ASSOCIATIONS AND INSTITUTIONS, 2012).
} 
passos básicos do planejamento devem ser: estabilizar os efeitos do desastre, avaliar os danos e iniciar os esforços de recuperação (FARMER, 2012).

\subsubsection{Avaliar}

Como os recursos eletrônicos requerem grande parte dos orçamentos das bibliotecas, as práticas voltadas ao desenvolvimento de coleções tornaram-se amplamente focadas na avaliação utilizando para isso métodos que se baseiam em dados para justificar os gastos e verificar se os recursos estão sendo investidos com sabedoria (VERMINSKI; BLANCHAT, 2017). Uma vez que o recurso está disponibilizado é importante continuar a avaliá-lo ao longo do tempo, para obter um diagnóstico útil é imperativo medir diferentes recursos ao longo do tempo (HOSBURGH, 2014).

Além do custo e do uso, que podem ser medidos com base nos dados estatísticos, Farmer (2009) salienta ser necessário ainda verificar questões como o feedback da comunidade acadêmica. É interessante perguntar aos usuários se suas necessidades estão sendo atendidas com um recurso eletrônico específico (HOSBURGH, 2014). Karmakar (2015) complementa que para verificar o valor de determinada fonte de informação a biblioteca deveria obter estatísticas de uso e informações dos professores, funcionários e usuários finais.

Para que se possa compreender o quanto os recursos estão sendo utilizados e o quão econômico ele é, se comparado a outros produtos (INTERNATIONAL FEDERATION OF LIBRARY ASSOCIATIONS AND INSTITUTIONS, 2012), os relatórios de estatísticas de uso fornecidos pelo fornecedor são um instrumento importante para decidir a respeito da continuidade ou da interrupção de tal investimento (VERMINSKI; BLANCHAT, 2017). Embora, Montgomery e Sparks (2000) destaquem alguns dos problemas encontrados nas estatísticas de uso recebidas dos fornecedores, como, por exemplo, a ausência de padrão, falta de regularidade e atrasos na entrega, terminologia utilizada não padronizada, intervalos diferentes e possibilidade de perda dos dados em caso de avaria no sistema.

Uma alternativa aos problemas mencionados é a adoção de padrões reconhecidos internacionalmente, tais como: Guidelines for Statistical Measures of Usage of Web- 
Based Information Resources ${ }^{6}$ do ICOLC, o Code of Practice do COUNTER (Counting

Online Usage of Networked E-resources) ${ }^{7}$ e/ou o SUSHI (Standard Usage Statistics Harvesting Initiative) ${ }^{8}$ (INTERNATLIONAL FEDERATION OF LIBRARY ASSOCIATIONS AND INSTITUTIONS, 2012).

Hosburgh (2014) indica o uso de estatísticas baseadas em COUNTER para medição padronizada de periódicos, bancos de dados, livros eletrônicos e outros recursos. Para o autor as estatísticas do COUNTER são muito importantes para tomar decisões com base em informação e, principalmente, no momento da renovação.

Entretanto, não basta que o fornecedor ofereça estatísticas padronizadas, é necessário ainda que o bibliotecário possua o conhecimento destas ferramentas, padrões e iniciativas para que saiba utilizá-las e interpretar os dados (NORTH AMERICAN SERIALS INTEREST GROUP, 2016).

Além dos dados fornecidos pelos relatórios, como relação custo/benefício, quantitativo de uso e número de acesso, Emery e Stone (2013) citam ainda outros fatores que devem ser levados em conta na avaliação, como por exemplo, alterações nas licenças, análise de sobreposição com outros recursos, aumento do preço percentual, valor da taxa de câmbio, mudanças na cobertura do conteúdo, DRM, complicações no fornecimento do serviço (faturamento atrasado ou problemas de plataforma), títulos transferidos e adicionados, entre outros.

Somam-se as problemáticas na avaliação das coleções levantadas pelos autores, ainda a exaustividade do trabalho a ser realizado, visto que todas as informações e relatórios mencionados devem ser coletados e todos os dados levantados precisam ser organizados para serem analisados. Necessárias ainda são a agilidade e objetividade nestes levantamentos, visto que qualquer equívoco pode desencadear em inconsistências ou atrasos nas decisões a serem tomadas, podendo inclusive, deixar a comunidade sem a informação que necessita.

\footnotetext{
${ }^{6}$ Diretrizes para estatísticas de uso de recursos de informações baseadas na Web. Tem como objetivo definir o conteúdo e a forma de dados estatísticos sobre o uso de recursos eletrônicos (INTERNATIONAL FEDERATION OF LIBRARY ASSOCIATIONS AND INSTITUTIONS, 2012).

${ }^{7}$ Contador de Estatísticas de uso conforme (Contando o uso online de recursos eletrônicos em rede). É um protocolo que permite aos editores e fornecedores apresentar os dados de forma clara e consistente em todos os seus produtos (INTERNATIONAL FEDERATION OF LIBRARY ASSOCIATIONS AND INSTITUTIONS, 2012).

${ }^{8}$ Iniciativa de Coleta de Estatísticas de Uso Padrão. Este padrão, define um modelo automatizado de solicitação e resposta para coleta de dados de uso de recursos eletrônicos, projetado como um protocolo generalizado extensível a vários relatórios de uso, inclusive o COUNTER (NATIONAL INFORMATION STANDARDS ORGANIZATION, 2014).
} 
Nesse sentido, a avaliação do recurso eletrônico respalda a decisão sobre renovações ou cancelamentos e substituição do recurso (HOSBURGH, 2014). Em relação à renovação, Hosburgh (2014) destaca que o cronograma de renovação é um dos aspectos mais importantes da revisão anual, na qual é desejável verificar o aumento do preço em relação ao ano anterior bem como analisar os dados coletados e, ainda, recomenda uma abordagem mais pró ativa no contato com o fornecedor antes de receber a fatura de renovação, para que os custos e quaisquer condições possam ser revistos.

Como nem toda a avaliação pode ser positiva, os bibliotecários devem estar preparados para lidar com possíveis cancelamentos ou revisões para substituição do recurso. Nesses casos, torna-se relevante reavaliar as fontes de informações disponíveis no mercado, com a intenção de identificar recursos mais vantajosos para a comunidade, afirma Hosburgh (2014). O autor ainda complementa que uma conversa sobre cancelamento com o fornecedor pode abrir portas para negociações, sendo que se for necessária uma nova licença, esta deve passar por uma revisão.

Importante assim, que as bibliotecas mantenham um calendário anual de avaliação dos recursos eletrônicos de informação que disponibilizam, considerando sempre as características da instituição mantenedora, do seu público alvo e o alinhamento com os recursos financeiros disponíveis.

\section{CONSIDERAÇÕES FINAIS}

Com a elaboração da proposta do modelo conceitual do ciclo de vida dos recursos eletrônicos, percebeu-se que os autores são unanimes em afirmar que o ciclo de vida dos recursos eletrônicos vem alterando o fluxo de trabalho nas bibliotecas.

Apesar disso, também se constatou que os autores delineiam diferentes etapas para o ciclo e, portanto, distinguem o fluxo de trabalho acerca dos recursos eletrônicos, sendo que as primeiras questões voltadas para pensar um sistema de gerenciamento de recursos eletrônicos foram levantadas por Jewell, em 2001, apesar do primeiro desenho de ciclo de vida ser apresentado por Pesch, em 2004.

Percebe-se ainda que os únicos autores que fazem menção ao processo de selecionar foram Jewell (2001) e Sadeh e Ellingsen (2005) que incluem como uma das etapas do ciclo e, Farmer (2009), que menciona as políticas de seleção como parte da 
etapa de Pré-Planejamento. Desta forma, percebe-se que a atividade de tomada de decisão, inerente a profissão dos bibliotecários, tem ficado subjugada na era das coleções eletrônicas. Por isso, utilizou-se o documento da International Federation of Library Associations and Institutions (2012) para reforçar no ciclo proposto, com mais propriedade para as questões inerentes a seleção, pois esta atividade é compreendida como essencial para a formação de uma coleção condizente com a necessidade informacional dos usuários da biblioteca.

A respeito das diferentes visões sobre as etapas do ciclo, salienta-se que autores com Sadeh e Ellingsen (2005) e Emery e Stone (2013) entendem que o fluxo inicia com a descoberta ou investigação de conteúdos, enquanto para Jewell (2004) a primeira etapa é a seleção, mencionada por Farmer (2009) como um item do pré-planejamento e considerada pela International Federation of Library Associations and Institutions (2012) como uma questão chave para o desenvolvimento de coleções eletrônicas; ainda para Pesch $(2004,2008)$ e Karmakar (2015), o ciclo inicia com a aquisição. Destaca-se ainda mais algumas questões relativas as etapas do ciclo, como o provimento do acesso, visto por Hosburgh (2014) como uma etapa da implementação, sendo que para Pesch (2004,2008) e Karmakar (2015) este item destaca-se como uma etapa completa; diferenças nos termos utilizados, porém usados no mesmo sentido, como por exemplo investigar para Hosburgh (2014) e descobrir/identificar, utilizado por Anbu K., Kataria e Ram (2013). Outro ponto observado foi que, apesar de alguns autores, como Pesch (2004, 2008) e Karmakar (2015) utilizarem como uma etapa do ciclo, administrar os recursos, optou-se pela utilização do termo gerenciar, por entender que se aplica melhor ao contexto.

Apesar dos autores descreverem diferentes etapas para o ciclo e que nem todas as atividades estão especificadas em todos os ciclos propostos na literatura, quando presentes, apresentaram concordância com relação aos comentários e posturas profissionais.

Constatou-se ainda a ausência de publicações brasileiras que tratam a respeito do ciclo de vida dos recursos eletrônicos de informação. Desse modo, o modelo conceitual proposto foi elaborado tomando-se por base a literatura consultada e a realidade das bibliotecas universitárias brasileiras observadas sob a óptica das autoras.

Destaca-se ainda que, a biblioteca como uma instituição historicamente reconhecida no apoio à pesquisa e nas demais atividades desenvolvidas na universidade, 
mantém seu papel na estrutura do desenvolvimento científico, e por isso, salienta-se que, embora sua atividade fim não tenha sido alterada substancialmente na era das informações eletrônicas, suas atividades meio necessitaram de readequações incluindo a incorporação de novos conhecimentos, inclusive a respeito do ciclo de vida dos recursos eletrônicos de informação, bem como de áreas distintas como as tecnológicas e legais. 


\section{REFERÊNCIAS}

ANBU K., John Paul; KATARIA, Sanjay; RAM, Shri. Dynamics of managing electronic resources: electronic resource management system (ERMS) initiatives. Journal of Library \& Information Technology, v. 33, n. 4, p. 300-305, jul. 2013.

BARATIN, Marc; JACOB, Christian. O poder das bibliotecas: a memória dos livros no Ocidente. 2. ed. Rio de Janeiro: UFRJ, 2006.

BUENO DE LA FUENTE, Gema. Modelo de repositorio institucional de contenido educativo (RICE): la gestión de materiales digitales de docencia y aprendizaje en la biblioteca universitária. Getafe, 2010. Tese (Doutorado) - Universidad Carlos III de Madrid, Departamento de Biblioteconomia y Documentación. Disponível em: $<$ https:/earchivo.uc3m.es/handle/10016/9154>. Acesso em: 04 jun. 2018.

CRESWELL, John W. Projeto de pesquisa: métodos qualitative, quantitative e misto.2. ed. Porto Alegre: Artmed, 2007.

EMERY, Jill; STONE, Graham. Techniques for Electronic Resource Management. Library Technology Reports, v. 49, n. 2, 2013.

FARMER Lesley. S. J. The life cycle of digital reference sources. In: PISARCHIK, Alexander N.; SHANG, Yilun; BULUCEA, Cornelia Aida. Recent Advances in Communications, Circuits and Technological Innovation. Paris: WSEAS, 2012. p. 99102.

$<$ http://www.wseas.org/multimedia/books/2012/Paris/CICOCOM.pdf $>$. Acesso em: 15 jun. 2018.

FARMER, Lesley. S. J. The life cycle of digital reference sources. The Reference Librarian, v.50, n. 2, 2009, p. 117-136. Disponível em: $<$ https://www.tandfonline.com/doi/abs/10.1080/02763870902755957>. Acesso em: 28 abr. 2018.

GEUTHER, Christina M. Challenges of the electronic resources life cycle and practical ways to overcome them. Kansas Library Association College and University Libraries Section Proceedings, v.7, n. 1, 2017.

GIL, Antônio Carlos. Como elaborar projetos de pesquisa. 5. ed. São Paulo: Atlas, 2010 .

HAHN, Karla L. SERU (Shared Electronic Resource Understanding): opening up new possibilities for electronic resource transactions. D-Lib Magazine, v. 13, n. 11/12, nov./dez. 2007.

HOSBURGH, Nathan. Managing the electronic resources lifecycle: creating a comprehensive checklist using techniques for electronic resource management (TERMS). The Serials Librarian, v. 66, p. 212-219, 2014. 
INTERNATIONAL FEDERATION OF LIBRARY ASSOCIATIONS AND INSTITUTIONS. Questões chave para o desenvolvimento de coleções de recursos eletrónicos: um guia para bibliotecas. Secção de Aquisições e Desenvolvimento de Coleções, ago. 2012. Disponível em: <https://www.ifla.org/files/assets/acquisitioncollection-development/publications/electronic-resource-guide-pt.pdf $>$. Acesso em: 15 maio 2018.

JEWELL, Timothy D. Selection and presentation of commercially avaiable electronic resources: issues and practices. Washington: Council on Library and Information Resources, 2001.

JOHNSON, Peggy. Fundamentals of collection development \& management. 3 rd ed. Chicago: ALA, 2014.

KARMAKAR, Ranjan. Electronic resource management (ERM) - life cycle of an eresource: an evaluative study. Research Spectra. v. 1, n.1, abril 2015, p. 39-46.

LIBLICENSE: licensing digital content. Chicago: Center for Research Libraries, 2015. Disponível em: $<$ http://liblicense.crl.edu/licensing-information/model-license/>. Acesso em: 15 ago. 2018.

MARCONI, Marina de Andrade; LAKATOS, Eva Lakatos. Fundamentos de metodologia científica. 7. ed. São Paulo: Atlas, 2010.

MARTINS, Wilson. A palavra escrita: história do livro, da imprensa e da biblioteca. 3. ed. rev. e atual. São Paulo: Ática, 2002.

MCGARRY, Kevin. O contexto dinâmico da informação: uma análise introdutória. Brasília, DF: Lemos Informação e Comunicação, 1999.

MINCHEW, Tessa. SLUTSKAYA, Sofia. Tha path of least resistance: using available tools do support the e-resource lifecycle. The Serials Librarian, v. 70, p. 236-246, 2016.

MONTGOMERY, Carol Hansen; SPARKS, JoAnne L. The transition to an electronic journal collection: managing the organizational changes. Serials review, v. 26, n. 3, p. 418, 2000.2 Disponível em: $<$ https://www.tandfonline.com/doi/abs/10.1080/00987913.2000.10764596? src=recsys\& journalCode $=$ usrv20>. Acesso em: 10 maio 2018 .

NORTH AMERICAN SERIALS INTEREST GROUP. Core competencies for electronic resources librarians: final version. New York: NASIG, 2016. Disponível em: $<\mathrm{http}$ //www.nasig.org/site_page.cfm?pk_association_webpage_menu=310\&pk_associa tion_webpage $=7802>$. Acesso em: 22 maio 2018.

NATIONAL INFORMATION STANDARDS ORGANIZATION. ANSI/NISO Z39.932014: The Standardized Usage Statistics Harvesting Initiative (SUSHI) Protocol. Baltimore: NISO, 2014. Disponível em: $<$ http://groups.niso.org/apps/group_public/download.php/14217/Z39-93-2014_SUSHI1_7.pdf $>$. Acesso em: 21 maio 2018. 
NATIONAL INFORMATION STANDARDS ORGANIZATION. NISO RP-7-2012: SERU: A Shared Electronic Resource Understanting. Baltimore: NISO, 2012. Disponivel em: $\quad<$ https://groups.niso.org/apps/group_public/download.php/8593/RP-72012_SERU.pdf $>$. Acesso em: 19 maio 2018.

NUNES, Martha Suzana Cabral; CARVALHO, Kátia de. As bibliotecas universitárias em perspectivas históricas: a caminho do desenvolvimento durável. Perspectivas em Ciência da Informação, v. 21, n. 1, p. 173-193, jan./mar. 2016. Disponível em: $<$ http://portaldeperiodicos.eci.ufmg.br/index.php/pci/article/view/2572/1708>. Acesso em: 31 jul. 2017.

PÉREZ ALARCÓN, Adoració. La gestión de los recursos digitales en una biblioteca virtual: la biblioteca virtual de la UOC. In: COLÓQUIO INTERNACIONAL DE CIENCIAS DE LA DOCUMENTACIÓN, 2., 2001. Salamanca, Anais... Salamanca: Biblioteca Virtual de la UOC, 2001.

PESCH, Oliver. Library standards and e-resource management: a survey of current initiatives and standards efforts. The Serials Librarian, v. 55, n. 3, p. 481-486, 2008.

PESCH, Oliver. Life-cycle of an e-resources. In: ASSOCIATION FOR LIBRARY COLLECTIONS \& TECHNICAL SERVICES ANNUAL CONFERENCE, 2004. Orlando. ALCTS presentations and materials from ALA Annual Conferences... Chicago: American Library Association, 2004. Slides. Disponível em: $<$ http://www.ala.org/alcts/sites/ala.org.alcts/files/content/events/pastala/annual/04/Pesch .pdf>. Acesso em: 02 maio 2018.

REDMOND, D. A.; SINCLAIR, M. P.; BROWN, E. University libraries and university research. College \& Research Libraries, v. 33, n. 6, nov. 1972.

ROTHER, Edna Terezinha. Revisão sistemática x revisão narrativa. Acta Paulista de Enfermagem, São Paulo , v. 20, n. 2, p. v-vi, jun. 2007. Disponível em: $<$ http://dx.doi..rg/10.1590/S0103-21002007000200001>. Acesso em: 21 jul. 2018.

SAN JOSÉ MONTANO, Blanca. La gestión de la colección cooperativa en las bibliotecas universitarias a comienzos del siglo XXI. Madrid, 2011. Tese (Doutorado) - Universidad Carlos III de Madrid, Departamento de Biblioteconomia y Documentación. Disponível em: <https://e-archivo.uc3m.es/handle/10016/10412>. Acesso em: 28 abr. 2018.

VERGUEIRO, Waldomiro de Castro Santos. Desenvolvimento de coleções: uma nova visão para o planejamento de recursos informacionais. Ciência da Informação, Brasília, v. 22, n. 11, p. 13-21. jan./abr. 1993. Disponível em: $<$ http://revista.ibict.br/index.php/ciinf/article/view/512>. Acesso em: 12 ago. 2017.

VERGUEIRO, Waldomiro de Castro Santos. Desenvolvimento de coleções. São Paulo: Polis: Associação Paulista de Bibliotecários, 1989.

VERMINSKI, Alana; BLANCHAT, Kelly Marie. Fundamentals of electronic resources management. Chicago: Neal-Schuman, 2017. 
WEITZEL, Simone da Rocha. Elaboração de uma política de desenvolvimento de coleções em bibliotecas universitárias. 2. ed. Rio de Janeiro: Interciência: Intertexto, 2013.

WEITZEL, Simone da Rocha. O desenvolvimento de coleções e a organização do conhecimento: suas origens e desafios. Perspectivas em Ciência da Informação, Belo Horizonte, v.7, n.1, p. 61-67, jan./jun. 2002. 